\title{
INTEGRAL REPRESENTATION THEOREMS IN PARTIALLY ORDERED VECTOR SPACES
}

\author{
PANAIOTIS K. PAVLAKOS
}

(Received 21 July 1989)

Communicated by S. Yamamuro

\begin{abstract}
Defining a Radon-type integration process we extend the Alexandroff, Fichtengolts-KantorovichHildebrandt and Riesz integral representation theorems in partially ordered vector spaces.

We also identify some classes of operators with other classes of operator-valued set functions, the correspondence between operator and operator-valued set function being given by integration.

All these established results can be immediately applied in $C^{*}$-algebras (especially in $W^{*}$ algebras and $A W^{*}$-algebras of type $\mathrm{I}$ ), in Jordan algebras, in partially ordered involutory $\left(O^{*}\right.$-)algebras, in semifields, in quantum probability theory, as well as in the operator FeynmanKac formula.
\end{abstract}

1980 Mathematics subject classification (Amer. Math. Soc.) (1985 Revision): 28 B 15; secondary 28 B 05, 46 G 10.

\section{Introduction}

The main object of this paper is to characterize the Alexandroff, FichtengoltsKantorovich-Hildebrandt and Riesz celebrated integral representation theorems, in partially ordered vector spaces.

The basic tool used in this approach is a good notion of a Radon-type integration process based on the order structure of the corresponding vector spaces.

For simplicity we confine our attention to finite partially ordered vectorvalued $\sigma$-measures (respectively measures) and uniform order convergence,

(C) 1991 Australian Mathematical Society $0263-6115 / 91 \$ A 2.00+0.00$ 
but our process can be easily developed in the more general setting of locally finite partially ordered group-valued $\sigma$-meausres (respectively measures) and uniform order convergence almost everywhere, thus extending our results in [50].

Following this pattern our results generalize previous ones of S. Bochner [9], R. R. Christian [15], J. D. M. Wright [76-79] and S. Khurana [41], who established their results in integrable spaces of real or complex-valued functions with respect to partially ordered vector-valued $\sigma$-measures or measures, as well as of A. W. Wickstead [75] and R. Cristescu [17], who also defined types of integrability concerning vector lattice-valued functions with respect to vector lattice-valued $\sigma$-measures.

In the construction of our integral, in contrast to various concepts of vector integrals in topological vector spaces of R. G. Bartle, N. Dunford and J. Schwartz [5], R. G. Bartle [6], J. Batt and E. J. Berg [7], J. K. Brooks and P. W. Lewis [12], J. K. Brooks and N. Dinculeanu [13], N. Dinculeanu [22], I. Dobrakov [25, 26], N. Dunford [27], R. J. Easton and D. H. Tucker [28], J. R. Edwards and S. G. Wayment [31], R. K. Goodrich [35], A. Shuchat [64], K. Swong [68] and D. J. Uherka [72], there are no finiteness restrictions on variation or semivariation (respectively Gowurin, in terminology of [28], [31], [35], [72] of the corresponding vector $\sigma$-measures (respectively measures)).

On the other hand, J. J. Diestel and J. J. Uhl [21], N. Dinculeanu [22] and A. Shuchat [64], have shown the existence of Banach lattice valued positive $\sigma$ measures (respectively measures) with unbounded variation or semivariation.

As has been indicated in [21], even if the vector $\sigma$-measure (respectively measure) is real-valued, it need not to be of bounded variation or semivariation.

Thus covering another aspect of the problem of integration of vectorvalued functions with respect to vector-valued $\sigma$-measures (respectively measures), we obtain integral representations analogous to those of the authors mentioned previously.

In particular the examined cases under considerations give extensions of the crucial concept of Radon measure in the sense of $\mathbf{N}$. Bourbaki [11].

For other extensions of this very interesting concept of Radon measure (in the sense of N. Bourbaki) see also R. Cristescu $[16,17]$ and L. N. Tsitsas [71].

A $\sigma$-measure (respectively measure) on a $\sigma$-algebra $H_{\sigma}(T)$ (respectively algebra $H(T)$ ) of subsets of a space $T$ with values in a partially ordered vector space (p.o.v.s) $Y$, is a positive $\sigma$-additive with respect to order convergence (respectively finitely additive) set function $m: H_{\sigma}(T) \rightarrow Y$ (respectively $m: H(T) \rightarrow Y$ ) . 
The defined integral has the form $\int_{T} f(t) d m(t)$, where the function $f$ is defined on $T$ and takes values in a second p.o.v.s. $X$. The integral has values in the Dedekind completion $\widehat{V}$ of a third p.o.v.s. $V$ via a positive bilinear separately order continuous function from $X \times Y$ into a forth p.o.v.s. $Z$ (denoted by “.").

In Section 2 we summarize some general definitions and preliminary results which will be needed for the later development.

In Section 3 we define the p.o.v.s. $M(T, X)$ (respectively $M_{t}(T, X)$ ) of $H_{\sigma}(T)$-measurable (respectively totally $H(T)$-measurable) functions from $T$ into $X$ and derive some approximation-type results in those spaces.

Next (Section 4) we develop an integration process for functions in $M(T, X)$ (respectively $M_{t}(T, X)$ ) with respect to a $\sigma$-measure (respectively measure) $m$ and establish the continuity of this integral with respect to uniform order convergence on the p.o.v.s. $\mathscr{L}^{1}(T, X)$ (respectively $M_{t}(T, X)$ ) of $H_{\sigma}(T)$-integrable (respectively totally $H(T)$-measurable) functions, which contains the $H_{\sigma}(T)$-elementary integrable (respectively $H(T)$-simple) functions.

It is worth noting that S. Mazur and W. Orlicz [46] and D. PrzeworskaRolewicz and S. Rolewicz [53] have shown that the Riemann, Lebesgue or Bochner integrals do not behave well in some metric linear lattices. So those integrals are not continuous for the topology of uniform convergence on the space of simple functions.

N. Dunford has defined in [27] a spectral integral which also has the surprising and unexpected property of being discontinuous with respect to the topology of uniform convergence on the space of simple functions.

Section 5 treats Fichtengolts, Kantorovich and Hildebrand type theorems for some classes of operators defined on the space $\mathscr{L}^{1}(T, X)$ (respectively $\left.M_{t}(T, X)\right)$ and having values in $Y$.

Up to this point we have considered only order structure on the vector spaces $X, Y, Z$ and no structure for the space $T$.

So in Sections 6, and 7, $T$ (respectively $X$ ) is endowed with Hausdorff (respectively norm) topologies and $Y, Z$ are p.o.v.s.

We compare notions of measurability with total boundness or continuity in $M(T, X)$ (respectively $M_{t}(T, X)$ ) .

We also examine relations between $\left(\tau_{u} o\right)$ - and $(u o)$-continuous linear operators with domain a subspace $Q(T, X)$ of $F(T, X)$ equipped with topology $\tau_{u}$ of uniform convergence and range $Y$.

We recall that classes of "topological-order" continuous linear operators have been extensively investigated in normed lattices by L. V. Kantorovich, B. Z. Vulikh and A. G. Pinsker in [37], [38], [74].

Further we examine two distinguished cases for $T$ and establish general 
forms of the following famous theorems in analysis.

The Alexandroff representation theorem (Section 8): If $T$ is a normal topological space, $U$ a positive $\left(\tau_{u} o\right)$-continuous linear operator with domain the partially ordered normed space $C_{t b}(T, X)$ of totally bounded continuous functions from $T$ into $X$ and with range $Y$ then

$$
U(f)=\int_{T} f(t) d m(t),
$$

for a unique operator-valued Borel measure $m$.

The Riesz representation theorem (Section 9): If $T$ is a locally compact topological space, $U$ a positive $\left(\tau_{u} o\right)$-continuous linear operator with domain the partially ordered normed space $C_{o o}(T, X)$ of continuous functions with compact support from $T$ into $X$ and with range $Y$ then $U(f)=$ $\int_{T} f(t) d m(t)$, for a unique operator-valued Borel $\sigma$-measure $m$.

We use these representation theorems to identify some classes of $\left(\tau_{u} o\right)$ continuous linear operators from $C_{t b}(T, X)$ (respectively $C_{o o}(T, X)$ ) into $Y$ with other classes of operator-valued Borel set functions, the correspondence between operator and set function being given by integration.

Next, if $X$ is a partially ordered normed algebra and $Y$ a partially ordered algebra, it makes sense to consider operators from $C_{t b}(T, X)$ (respectively $\left.C_{o o}(T, X)\right)$ into $Y$ which are not only $\left(\tau_{u} o\right)$-continuous and linear but which also preserve multiplication.

Section 10 is devoted to answering the natural question: how the additional property of these operators being multiplicative is reflected in the representing $\sigma$-measures (respectively measures)?

We conclude the paper with significant and important applications in $C^{*}$ algebras, especially in $W^{*}$-algebras and in $A W^{*}$-algebras of type $\mathrm{I}[10,18$, $23,24,55,80,81]$, in Jordan algebras $[2,32,65-67,70,82]$ in $O^{*}$-algebras $[59,61]$, in semifields $[3,44,45,56-58,60,62]$, in quantum probability theory $[14,18-20,29,30,36,51]$ as well as in the operator Feynman-Kac formula $[34,42,43]$.

The subject of $\sigma$-measures (respectively measures) and integrals taking values in partially ordered vector spaces of operators acting on a Hilbert space $H$ has received much attention in the recent mathematical and physics literature $[8,14,18-20,29,30,36,51,79]$.

Thus our results can be immediately applied to integrating operator-valued measurable functions with respect to positive operator-valued $\sigma$-measures (respectively measures) acting on $H$.

In particular the results of Section 10 answer the question when the representing $\sigma$-measures (respectively measures) are projection-valued.

On the other hand our results have important applications to semifieldvalued $\sigma$-measures (respectively measures) and integrals. 
This very interesting concept has been defined and studied by the Russian school of Sarymsakov, Benderskiì, Čilin, Rubšteǐn, Halikulov, Kučkarov and others in a series of papers $[44,45,56-58,60,62]$.

The class of semifields is much wider than the class of topological semifields [3], and it is contained in the class of $K_{\sigma}$-spaces.

As is well known, many attempts have been made to use the setting of p.o.v.s. in the theory of the mathematical foundation of quantum mechanics (see, for example, [36]).

To this end we describe in Section 11 how the presented results can be directly applied to a state space of quantum mechanics, integrating vectorvalued random variables in a sense similar to that of E. Rowecka [54] or H. Umegaki and A. T. Bharucha-Reid [73] with respect to an operator-valued instrument (respectively observable).

The fruitful concept of instruments has been introduced by E. B. Davies and J. T. Lewis in [20], generalizing that of observables in a statistical system of quantum probability theory. Accordingly there is a canonical procedure for associating an observable to an instrument. It is easily verified that an instrument (respectively observable) is a $\sigma$-measure (respectively measure) taking values in a certain partially ordered Banach space of linear operators.

On the other hand, the main problems in mathematical physics concerning a perturbation formula of the Feynman-Kac type for the solution semigroups of some initial-value problems lead to the development of an integration theory which does not presuppose finite variation or semivariation of the corresponding measures (respectively $\sigma$-measures) (cf. [42, 43]).

The resulting notions and techniques (based on the partial ordering) can be immediately applied to express the superpositions of some semigroups of operators, including those which describe the motion of quantum mechanical particles in a potential force field.

Now another available application of our methods employed here seems to be in neutron diffusion theory. In that direction the reader may consult the excellent treatise of P. M. Morse and H. Feschbach [48].

We refer to $[1,10,22-24,34,37,52,55,63,69,74,83]$ for general references about p.o.v.s., measure theory, normed algebras and semigroups of linear operators.

\section{Notation and terminology}

We now give the basic properties of a p.o.v.s. that will be needed in the subsequent development.

Throughout this paper $X$ will denote a p.o.v.s. over the real field $\mathbb{R}$ with 
positive cone $X^{+}$. We call $X$ positively generated if $X=X^{+}-X^{+}$.

Let $\left(x_{\delta}\right)_{\delta \in \Delta}$ be a net in $X$ and $x \in X$. We say that $\left(x_{\delta}\right)_{\delta \in \Delta}$ is increasing (respectively decreasing) if $x_{\delta} \geq x_{\delta^{\prime}}$ (respectively $x_{\delta} \leq x_{\delta^{\prime}}$ ) whenever $\delta \geq$ $\delta^{\prime}$. The notation $x_{\delta} \uparrow x$ means that the net $\left(x_{\delta}\right)_{\delta \in \Delta}$ is increasing and $x=\sup \left\{x_{\delta}: \delta \in \Delta\right\}$, and similarly for $x_{\delta} \downarrow x$. We say that $\left(x_{\delta}\right)_{\delta \in \Delta}$ order converges to $x \quad\left(x_{\delta} \stackrel{0}{\rightarrow} x\right.$ or $o$-lim $\left.x_{\delta}=x\right)$ if there exists a decreasing net $\left(y_{\delta}\right)_{\delta \in \Delta}$ such that $y_{\delta} \downarrow 0$ and $-y_{\delta} \leq x_{\delta}-x \leq y_{\delta}$, for all $\delta \in \Delta$. Recall that this kind of order convergence does not in general coincide with that defined in [50], even if $X$ is a vector lattice [49]. For details about various other concepts of order convergence see [47] and [49].

We call $X$ almost Archimedean (respectively Archimedean) if $-\varepsilon y \leq$ $x \leq \varepsilon y$ (respectively $\varepsilon x \leq y$ ) for some $y \in X^{+}$and all real numbers $\varepsilon>0$ implies $x=0$ (respectively $x \leq 0$ ).

We call $X$ monotone complete (respectively $\sigma$-complete) if every majorised increasing net (respectively sequence) in $X$ has a supremum in $X$. We say that $X$ has the diagonal property if whenever $\left\{x_{m, n}:(m, n) \in\right.$ $\mathbb{N} \times \mathbb{N}\} \subseteq X$ with $o-\lim _{n} x_{m, n}=x_{m} \in X$, for each $m \in \mathbb{N}$ and if $o-\lim x_{m}=x$ then there exists a strictly increasing sequence $\left\{n_{m}: m \in \mathbb{N}\right\} \subseteq \mathbb{N}$ such that $o-\lim x_{m, n_{m}}=x$.

Let $Y$ be another p.o.v.s. A linear operator $F: X \rightarrow Y$ is order (respectively $\sigma$-order) continuous if $F\left(x_{\delta}\right) \stackrel{o}{\rightarrow} 0$ (respectively $F\left(x_{n}\right) \stackrel{o}{\rightarrow} 0$ ) whenever $\left(x_{\delta}\right)_{\delta \in \Delta}$ (respectively $\left(x_{n}\right)_{n \in \mathbb{N}}$ ) is a net (respectively sequence) in $X$ such that $x_{\delta} \stackrel{o}{\rightarrow} 0$ (respectively $x_{n} \stackrel{o}{\rightarrow} 0$ ).

We denote by $L_{r}(X, Y)$ the p.o.v.s. of regular operators from $X$ into $Y$. By definition,

$L_{r}(X, Y):=\left\{F: X \rightarrow Y:\right.$ there exist positive linear operators $F_{i}: X \rightarrow Y$,

$$
i=1,2 \text { with } F=F_{1}-F_{2} \text {. }
$$

Also we put

$$
\begin{aligned}
& L_{\gamma}(X, Y)\left(\text { respectively } L_{\sigma}(X, Y)\right) \\
& \quad:=\{F: X \rightarrow Y: \text { there exist positive order (respectively } \sigma \text {-order) } \\
& \quad \text { continuous linear operators } F_{i}: X \rightarrow Y, \\
& \left.\qquad i=1,2 \text { with } F=F_{1}=F_{2}\right\} .
\end{aligned}
$$

In particular we write $X^{\sim}, X_{\gamma}^{\sim}, X_{\sigma}^{\sim}$ for $L_{r}(X, R), L_{\gamma}(X, R), L_{\sigma}(X, R)$.

Let $Z$ be a p.o.v. subspace of $X^{\sim}$ separating points of the positively generated p.o.v.s. $X$. Then the canonical map $i: X \rightarrow i(X) \subseteq Z^{\sim}, x \mapsto$ $i(x):=F_{x}\left(F_{x}(f)=f(x)\right.$, for every $\left.f \in Z\right)$ is an algebraic and order homomorphism of $X$ into the p.o.v. subspace $i(X)$ of $Z_{\gamma}^{\sim} \subseteq Z^{\sim}$. When 
$Z=X_{\gamma}^{\sim}$ (respectively $Z=X_{\sigma}^{\sim}$ ) $i$ also satisfies the following property: $0 \leq$ $i\left(x_{\delta}\right) \uparrow i(x)$ (respectively $0 \leq i\left(x_{n}\right) \uparrow i(x)$ ), whenever $\left(x_{\delta}\right)_{\delta \in \Delta}$ (respectively $\left.\left(x_{n}\right)_{n \in \mathbb{N}}\right)$ is a net (respectively sequence) in $X$ with $0 \leq x_{\delta} \uparrow x$ (respectively $\left.0 \leq x_{n} \uparrow x\right), x \in X$. In this case we identify $X$ with its image $i(X)$ and so $X$ is considered as a p.o.v. subspace of $Z_{\gamma}^{\sim}$. We call $X$ order reflexive (or perfect) if $Z=X_{\gamma}^{\sim}$ and the embedding $i$ is onto, that is, when $X=\left(X_{\gamma}^{\sim}\right)_{\gamma}^{\sim}$.

As is well known, even if $X$ is a Banach lattice the concepts of topological reflexivity and order reflexivity are not equivalent.

If $U \in L_{r}(X, Y)$ the restriction of the algebraic dual $U^{\sharp}$ of $U$ to $Y^{\sim}$ will be denoted by $U^{\sim}$ and is called the order adjoint of $U$. Clearly $U^{\sim} \in$ $L_{\gamma}\left(Y^{\sim}, X^{\sim}\right)$.

Also it is easily verified that if $X, Y$ are positively generated and $U \in$ $L_{\gamma}(X, Y)$ (respectively $U \in L_{\sigma}(X, Y)$ ) then the restriction $U^{\prime}$ of $U^{\sim}$ to $Y_{\gamma}^{\sim}$ (respectively $Y_{\sigma}^{\sim}$ ) maps $Y_{\gamma}^{\sim}$ (respectively $Y_{\sigma}^{\sim}$ ) into $X_{\gamma}^{\sim}$ (respectively $\left.X_{\sigma}^{\sim}\right)$. In particular $U \in L_{r}(X, Y)$ implies $\left(U^{\prime}\right)^{\prime}:=U^{\prime \prime} \in L_{\gamma}\left(\left(X^{\sim}\right)_{\gamma}^{\sim},\left(Y_{\gamma}\right)_{\gamma}^{\sim}\right)$ and if $Y_{\gamma}^{\sim}$ separates points of $Y$ then $U^{\prime \prime}\left(x^{\prime \prime}\right)=(U(x))^{\prime \prime}$ with $x^{\prime \prime}$ the image of $x$ under the canonical map of $X$ into $\left(X^{\sim}\right)_{\gamma}^{\sim}$.

The following simple facts concerning "order duals" of p.o.v.s. will prove to be useful later.

If $X$ is positively generated and $Y$ monotone complete (respectively $\sigma$-complete) then $L_{r}(X, Y), L_{\gamma}(X, Y)$ (respectively $L_{\sigma}(X, Y)$ ) are also monotone complete (respectively $\sigma$-complete).

A Dedekind completion for $X$ is a pair $(j, \widehat{X})$, where $\widehat{X}$ is a Dedekind complete vector lattice and $j: X \rightarrow \widehat{X}$ is a linear injection with the properties

(a) for each $x \in X, x \geq 0$ if and only if $j(x) \geq 0$ ( $j$ is bipositive);

(b) $\{j(x): x \in X$ with $j(x) \leq \hat{y}\} \neq \varnothing$ and $\hat{y}=\sup \{j(x): x \in X$ with $j(x) \leq \hat{y}\}$ for every $\hat{y} \in \hat{X}$;

(c) $j(x)=\sup \left\{j\left(x_{q}\right): q \in Q\right\}$ in $\hat{X}$, whenever $\left\{x_{q}: q \in Q\right\} \subseteq X$ with $x=\sup \left\{x_{q} ; q \in Q\right\}$ in $X$.

Moreover, a positively generated p.o.v.s. $X$ possesses a Dedekind completion if and only if $X$ is Archimedean. For a proof of this last result see [52, Proposition 1.19].

Next, let $X$ be a partially ordered algebra that is a positively generated p.o.v.s. which is at the same time an algebra such that $x \cdot y \geq 0$, whenever $x, y \in X$ with $x, y \geq 0$ and let $Z$ be a partially ordered subalgebra of $X^{\sim}$ separating points of $X$.

Suppose also that the multiplication in $X$ is separately order continuous. Then the canonical embedding $i: X \rightarrow i(X) \subseteq Z_{\gamma}^{\sim} \subseteq Z^{\sim}$ is an algebraic and order isomorphism of $X$ onto the partially ordered subalgebra $i(X)$ of $Z^{\sim}$ if we endow $Z^{\sim}$ with the following product, under which $Z^{\sim}$ becomes 
a partially ordered algebra. Given $F, G \in Z^{\sim}$, let $F \cdot G$ be defined by $(F \cdot G)(u)=F \cdot(G \cdot u), u \in Z$, where $(G \cdot u)(a):=G \cdot u_{a}$ and $u_{a}(x):=u(a \cdot x)$, for all $a, x \in X$.

\section{Partially ordered vector-valued measurable functions}

In this section $H_{\sigma}(T)$ (respectively $H(T)$ ) will denote a $\sigma$-algebra (respectively algebra) of subsets of $T$ and $X$ a positively generated p.o.v.s.

We also denote by $F(T, X)$ the p.o.v.s. of functions from $T$ into $X$, where the vector and ordering operation are defined pointwise. We identify $X$ with the subspace of $F(T, X)$ of all constant functions.

A net $\left(f_{\delta}\right)_{\delta \in \Delta}$ pointwise $o$-converges to $f \in F(T, X)$ on $T\left(p-\lim f_{\delta}=f\right.$ on $T$ ) if $o$-lim $f_{\delta}(t)=f(t)$, for every $t \in T$. It is well known that there exist p.o.v.s. $F(T, X)$ in which $o$-convergence does not imply $p$-convergence and conversely. However the following theorem can be easily verified.

THEOREM 3.1. Let $X$ be monotone complete (respectively $\sigma$-complete), $\left(f_{\delta}\right)_{\delta \in \Delta}$ (respectively $\left.\left(f_{n}\right)_{n \in N}\right)$ a net (respectively sequence) in $F(T, X)$ and $f \in F(T, X)$. The following assertions are equivalent:

(i) $o-\lim f_{\delta}=f$ (respectively $o-\lim f_{n}=f$ );

(ii) $p$-lim $f_{\delta}=f$ (respectively $p$ - $\lim f_{n}=f$ ) on $T$.

On the other hand, a net $\left(f_{\delta}\right)_{\delta \in \Delta}$ in $F(T, X)$ uniformly $o$-converges to $f \in F(T, X)$ on $T\left(u-\lim f_{\delta}=f\right.$ on $\left.T\right)$ if there exists a decreasing net $\left(u_{\delta}\right)_{\delta \in \Delta}$ in $X$ such that

$$
-u_{\delta} \leq f_{\delta}(t)-f(t) \leq u_{\delta}, \quad \text { for all }(\delta, t) \in \Delta \times T \text { and } u_{\delta} \downarrow 0 .
$$

Now let $E(T, X)$ (respectively $S(T, X)$ ) be the set of $H_{\sigma}(T)$-elementary (respectively $H(T)$-simple) measurable functions from $T$ into $X$. By definition,

$E(T, X):=\left\{f \in F(T, X):\right.$ there exists a countable partition $\left(A_{n}\right)_{n \in \mathbb{N}}$ of $T$ in $H_{\sigma}(T)$ such that $f(t)=x_{n}$, for every $\left.t \in A_{n}, x_{n} \in X, n \in N\right\}$ (respectively $S(T, X):=\{f \in F(T, X)$ : there exists a finite partition of $T$ in $H(T)$ such that $f(t)=x_{i}$, for every $\left.\left.t \in A_{i}, x_{i} \in X, i=1,2, \ldots, n\right\}\right)$.

Clearly, given $f \in E(T, X)$ there exists a sequence $\left(g_{n}\right)_{n \in \mathrm{N}}$ in $S(T, X)$ such that $p-\lim g_{n}=f$ on $T$.

Let $f \in F(T, X) ; f$ is $H_{\sigma}(T)$-measurable (respectively totally $H(T)$ measurable) if there exists a net $\left(f_{\delta}\right)_{\delta \in \Delta}$ in $E(T, X)$ (respectively $S(T, X)$ ) 
such that $u$-lim $f_{\delta}=f$ on $T$. This net will be called an approximating net for $f$.

We put

$$
M(T, X):=\left\{f \in F(T, X): f \text { is } H_{\sigma}(T) \text {-measurable }\right\}
$$

(respectively $M_{t}(T, X):=\{f \in F(T, X): f$ is totally $H(T)$-measurable $\}$ ). Clearly $M(T, X)$ (respectively $\left.M_{t}(T, X)\right), E(T, X)$ and $S(T, X)$ are p.o.v. subspaces of $F(T, X)$ with $S(T, X) \subseteq M_{t}(T, X), S(T, X) \subseteq$ $E(T, X) \subseteq M(T, X)$.

The following theorems concern the space $M(T, X)$, but similar results are also valid for the space $M_{t}(T, X)$.

The proof of the next theorem is similar to that of [50, Theorem 2.2] (recall that $X$ is of countable type if for every decreasing net $\left(x_{\delta}\right)_{\delta \in \Delta}$ in $X$ with $x_{\delta} \downarrow 0$ there exists an increasing sequence $\left\{\delta_{n}: n \in \mathbb{N}\right\} \subseteq \Delta$ such that $\left.x_{\delta_{n}} \downarrow 0\right)$.

THEOREM 3.2. Let $X$ be a vector lattice of countable type and $f \in M(T, X)$. Then there exists an increasing (respectively decreasing) sequence $\left(f_{n}\right)_{n \in \mathbb{N}}$ (respectively $\left.\left(g_{n}\right)_{n \in \mathrm{N}}\right)$ in $E(T, X)$ with $u-\lim f_{n}=f\left(\right.$ respectively $u-\lim g_{n}=$ f) on $T$.

TheOREM 3.3. Let $X$ be a p.o.v.s. with diagonal property and of countable type.

(i) If $\left(f_{\delta}\right)_{\delta \in \Delta}$ is a net in $M(T, X)$ such that $u-\lim f_{\delta}=f$ on $T$ then $f \in M(T, X)$.

(ii) For every $f \in M(T, X)$ there exists a sequence $\left(h_{n}\right)_{n \in N}$ in $S(T, X)$ such that $p-\lim h_{n}=f$ on $T$.

Proof. (i) This can be proved similarly to [50, Theorem 2.3(i)].

(ii) Let $f \in M(T, X)$. By definition there exists a sequence $\left(f_{n}\right)_{n \in \mathbb{N}}$ in $E(T, X)$ and $\left(x_{n}\right)_{n \in \mathbb{N}}$ in $X$ such that

$$
-x_{n} \leq f_{n}(t)-f(t) \leq x_{n} \text {, for all } n \in \mathbb{N} \text { and } t \in T \text {. }
$$

On the other hand, given $t \in T$ there exists $\left(y_{k, n}(t)\right)_{(k, n) \in \mathbb{N} \times \mathbf{N}}$ a double sequence in $X$ such that $y_{k, n}(t) \downarrow 0(k \rightarrow \infty, n \in \mathbb{N})$ and

$$
-y_{k, n}(t) \leq g_{k, n}(t)-f_{n}(t) \leq y_{k, n}(t),
$$

for all $(k, n, t) \in \mathbb{N} \times \mathbb{N} \times T$ with $\left(g_{k, n}\right)_{(k, n) \in \mathbb{N} \times \mathbb{N}}$ a double sequence in $S(T, X)$. 
Now by the diagonal property for every $t \in T$ there exists a sequence $\left(k_{n}(t)\right)_{n \in \mathbb{N}}$ in $\mathbb{N}\left(k_{n}(t) \leq k_{n+1}(t), n \in \mathbb{N}\right)$ such that

$$
y_{k_{n}(t), n}(t) \stackrel{o}{\rightarrow} 0 \quad \text { and } \quad-y_{k_{p}(t), p}(t) \leq g_{k_{p}(t), p}(t)-f_{p}(t) \leq y_{k_{p}(t), p}(t)
$$

for all $(p, t) \in \mathbb{N} \times T$. Hence $p$-lim $g_{n}=f$ on $T$ for the sequence $\left(g_{n}\right)_{n \in \mathbb{N}}$ in $S(T, X)$ defined by $g_{n}(t):=g_{k_{n}(t), n}(t), t \in T, n \in \mathbb{N}$.

\section{Integration}

In what follows $Z$ will denote a monotone complete p.o.v.s., $V$ the monotone complete (so Archimedean) p.o.v.s. ordered by the generating cone $Z^{+}\left(V=Z^{+}-Z^{+}\right)$and $\widehat{V}$ the Dedekind completion of $V$.

Assume also in this section that $X$ is positively generated and there exists a bilinear, positive and separately order continuous function from $X \times Y$ into $Z$ which we shall denote simply by juxtaposition.

Let $f \in E(T, X)$ (respectively $S(T, X)$ ). Then there exists a countable (respectively finite) partition $\left(A_{n}\right)_{n \in \mathbb{N}}$ (respectively $\left.\left(A_{i}\right)_{1 \leq i \leq n}\right)$ of $T$ by elements of the $\sigma$-algebra (respectively algebra) $H_{\sigma}(T)$ (respectively $H(T)$ ) such that $f(t)=a_{i}-b_{i}$, whenever $t \in A_{i}$ with $a_{i}, b_{i} \geq 0, i \in \mathbb{N}$ (respectively $i=1,2, \ldots, n)$.

Let also $m: H_{\sigma}(T) \rightarrow Y$ (respectively $m: H(T) \rightarrow Y$ ) be a $\sigma$-measure (respectively measure) on $H_{\sigma}(T)$ (respectively $H(T)$ ), that is, a positive and $\sigma$-additive (respectively finitely additive) with respect to order convergence in $Y$ set function on $H_{\sigma}(T)$ (respectively $H(T)$ ) with $m(\varnothing)=0$. Then $f$ is $H\left({ }_{\sigma}(T), m\right)$-integrable on $T$ if $o-\lim \sum_{i=1}^{n} a_{i} \cdot m\left(A_{i}\right)$ and $o-\lim \sum_{i=1}^{n} b_{i}$. $m\left(A_{i}\right)$ exist in $Z^{+}$. In this case we put $\int_{T} f(t) d m(t):=o-\lim \sum_{i=1}^{n}\left(a_{i}-b_{i}\right)$. $m\left(A_{i}\right)$, (respectively $\left.\int_{T} f(t) d m(t)=\sum_{i=1}^{n}\left(a_{i}-b_{i}\right) \cdot m\left(A_{i}\right)\right)$ and

$$
I(T, X):=\left\{f \in E(T, X): \text { there exists } \int_{T} f(t) d m(t)\right\} .
$$

Obviously, $I(T, X)$ is a p.o.v. subspace of $E(T, X)$ and $S(T, X) \subseteq$ $I(T, X)$.

It is not difficult to verify that the integral $\int_{T} f(t) d m(t)$ is well defined, is dependent only on $f$ and is independent of the particular way in which $f$ is written as an $H_{\sigma}(T)$-elementary (respectively $H(T)$-simple) function in $E(T, X)$ (respectively $S(T, X)$ ). Moreover it is independent of a rearrangement of the series $\left(\sum_{i=1}^{n}\left(a_{i}-b_{i}\right) \cdot m\left(A_{i}\right)\right)_{n \in \mathbb{N}}$.

We set $\int_{A} f(t) d m(t):=\int_{T} c_{A} f(t) d m(t)$, whenever $f \in I(T, X)$ (respectively $S(T, X)$ ) with $c_{A}$ the characteristic function of $A$ in $H_{\sigma}(T)$ 
(respectively $H(T)$ ) and $c_{A} f$ the $X$-valued function from $T$ defined by the formula $c_{A} f(t):=c_{A}(t) f(t), t \in T$.

The following theorem can be easily proved.

THEOREM 4.1. (i) The integral operator $I: I(T, X) \rightarrow V$ (respectively $I: S(T, X) \rightarrow V)$ with $I(f):=\int_{T} f(t) d m(t), f \in I(T, X)$ (respectively $S(T, X))$ is positive, linear and (uo)-continuous $\left(o-\lim I\left(f_{\delta}\right)=0\right.$, whenever $\left(f_{\delta}\right)_{\delta \in \Delta}$ is a net in $I(T, X)$ (respectively $S(T, X)$ ) with $u$-lim $f_{\delta}=0$ on $T)$.

(ii) $\int_{T} f(t) x d \mu(t)=\int_{T} f(t) d \mu_{x}(t)$, where $\mu: H_{\sigma}(T) \rightarrow X^{\sim}$ (respectively $\mu: H(T) \rightarrow X^{\sim}$ ) is a $\sigma$-measure (respectively measure) and $\mu_{x}: H_{\sigma}(T)$ $\rightarrow \mathbb{R}$ (respectively $\mu_{x}: H(T) \rightarrow \mathbb{R}$ ) with $\mu_{x}(A):=\mu(A)(x)$, whenever $A \in$ $H_{\sigma}(T)$ (respectively $A \in H(T)$ ), $f \in I(T, R)$ (respectively $f \in S(T, R)$ ), $x \in X^{+}$.

(iii) The set function $v: H_{\sigma}(T) \rightarrow V$ (respectively $w: H(T) \rightarrow V$ ) with $v(A):=\int_{A} f(t) d m(t)$ (respectively $w(A):=\int_{A} f(t) d m(t)$ ) for every $A \in$ $H_{\sigma}(T)$ (respectively $A \in H(T)$ ) is a $\sigma$-measure (respectively measure), whenever $f \in I(T, X)^{+}$with $f$ o-bounded (respectively $f \in S(T, X)^{+}$).

THEOREM 4.2. Let $\left(f_{\delta}\right)_{\delta \in \Delta}$ be an approximating net in $I(T, X)$ (respectively $S(T, X)$ ) of $f \in M(T, X)$ (respectively $f \in M_{t}(T, X)$ ). Then $u$-lim $\left(f_{\delta}-f_{\delta^{\prime}}\right)=0$ on $T$ and the net $\left(\int_{T} f_{\delta}(t) d m(t)\right)_{\delta \in \Delta}$ is o-fundamental in $V$.

Using similar arguments to those in the proof of [50, Theorem 3.10] we obtain a proof of the preceding statement.

TheOREM 4.3. Let $f \in M(T, X)$ (respectively $f \in M_{t}(T, X)$ ). Suppose that $\left(f_{\delta}\right)_{\delta \in \Delta}$ is an increasing approximating net in $I(T, X)$ (respectively $S(T, X)$ ) of $f$. Then there exists o-lim $\int_{T} f_{\delta}(t) d m(t)$ in $V$.

Proof. A proof is similar to that of [50, Theorem 3.11].

Corollary 4.4. Let $f \in M(T, X)$ (respectively $M_{T}(T, X)$ ). If $\left(f_{\delta}\right)_{\delta \in \Delta}$, $\left(g_{\theta}\right)_{\theta \in \Theta}$ are increasing approximating nets in $I(T, X)$ (respectively $S(T, X)$ ) of $f$, then $0-\lim \int_{T} f_{\delta}(t) d m(t)=o-\lim \int_{T} g_{\theta}(t) d m(t)$ in $V$.

THEOREM 4.5. Let $f \in M(T, X)$ (respectively $M_{t}(T, X)$ ) and $\left(f_{\delta}\right)_{\delta \in \Delta}$ an approximating net in $I(T, X)$ (respectively $S(T, X)$ ) of $f$. Then there exists the o-lim $\int_{T} f_{\delta}(t) d m(t)$ in the Dedekind completion $\widehat{V}$ of $V$. 
Proof. Recall that $V$ is Archimedean and may be identified with a.p.o.v. subspace of its Dedekind completion $\widehat{V}$. By Theorem $4.2\left(\int_{T} f_{\delta}(t) d m(t)\right)_{\delta \in \Delta}$ is $o$-fundamental in $V$ and hence $o$-converges in $\widehat{V}$.

CoRollary 4.6. Let $f \in M(T, X)$ (respectively $M_{t}(T, X)$ ). If $\left(f_{\delta}\right)_{\delta \in \Delta}$ and $\left(g_{\theta}\right)_{\theta \in \Theta}$ are approximating nets in $I(T, X)$ (respectively $S(T, X)$ ) of $f$, then $o-\lim \int_{T} f_{\delta}(t) d m(t)=o-\lim \int_{T} g_{\theta}(t) d m(t)$ in the Dedekind completion $\widehat{V}$ of $V$.

The preceding results lead to the following definitions. The $\left(H_{\sigma}(T), m\right)$ (respectively $(H(T), m)-$ ) integral of an element $f$ in $M(T, X)$ (respectively $\left.M_{t}(T, X)\right)$ is defined to be the element $\int_{T} f(t) d m(t)$ of $\widehat{V}$ with $\int_{T} f(t) d m(t):=o-\lim \int_{T} f_{\delta}(t) d m(t)$, where $\left(f_{\delta}\right)_{\delta \in \Delta}$ is an approximating net of $f$ in $I(T, X)$ (respectively $S(T, X)$ ). We also denote by $\mathscr{L}^{1}(T, X)$ the set of $\left(H_{\sigma}(T), m\right)$-integrable functions $f$ in $M(T, X)$. Clearly $\mathscr{L}^{1}(T, X)$ is a p.o.v. subspace of $M(T, X)$ and $M_{t}(T, x) \subseteq \mathscr{L}^{1}(T, X)$.

By Corollary 4.6, $\int_{T} f(t) d m(t)$ does not depend on the choice of an approximating net $\left(f_{\delta}\right)_{\delta \in \Delta}$ in $I(T, X)$ (respectively $S(T, X)$ ). Further we set $\int_{A} f(t) d m(t):=\int_{T} c_{A}(t) f(t) d m(t)$ whenever $f \in \mathscr{L}^{l}(T, X)$ (respectively $M_{t}(T, X)$ ) and $A \in H_{\sigma}(T)$ (respectively $A \in H(T)$ ).

On the other hand the propositions and theorems of the $\left(H_{\sigma}(T), m\right)$ (respectively $(H(T), m)$-) integral on the p.o.v.s. $I(T, X)$ (respectively $S(T, X)$ ) remain also valid for p.o.v.s. $\mathscr{L}^{1}(T, X)$ (respectively $M_{t}(T, X)$ ). In particular we state the following monotone-type convergence theorem. The proof is similar to that of [50, Theorem 3.13].

THEOREM 4.7. Let $\left(f_{n}\right)_{n \in \mathbb{N}}$ be a sequence in $\mathscr{L}^{1}(T, X)$ (respectively $\left.M_{t}(T, X)\right)$ such that $n \in \mathbb{N}$;

(i) there exists $x \in X$ with $0 \leq f_{n+1}(t) \leq f_{n}(t) \leq x$, for all $t \in T$,

(ii) there exists a sequence $\left(A_{n}\right)_{n \in \mathbb{N}}$ in $H_{\sigma}(T)$ (respectively $H(T)$ ) with $u$ - $\lim f_{n}=0$ on each $A_{n}, n \in \mathbb{N}$, and $o-\lim m\left(T-A_{n}\right)=0$;

(iii) $\widehat{V}$ has the diagonal property.

Then $o-\lim \int_{T} f_{n}(t) d m(t)=0$.

\section{Fichtengolts-Kantorovich-Hildebrandt-type theorems}

Let $X, Y$ be positively generated p.o.v.s. with $Y$ monotone complete. Recall that a linear operator $U: Q(T, X) \rightarrow Y$, with $Q(T, X)$ a p.o.v. 
subspace of $F(T, X)$, is (uo)-(respectively (po)- or (oo)-) continuous if $o-\lim U\left(f_{\delta}\right)=0$ whenever $\left(f_{\delta}\right)_{\delta \in \Delta}$ is a net in $Q(T, X)$ with $u$ - $\lim f_{\delta}=0$ on $T$ (respectively $p$ - $\lim f_{\delta}=0$ on $T$ or $o-\lim f_{\delta}=0$ ).

We denote by $L_{r}(Q(T, X), Y)_{u o}$ (respectively $L_{r}(Q(T, X), Y)_{p o}$ or $\left.L_{r}(Q(T, X), Y)_{o o}\right)$ the p.o.v.s. of all differences of positive (uo)-(respectively $(p o)$-or $(o o)$-) continuous linear operators from $Q(T, X)$ into $Y$.

THEOREM 5.1. Let $U: M_{t}(T, X) \rightarrow Y$ be a (uo)-continuous positive linear operator. Then there exists a unique measure $m: H(T) \rightarrow L_{r}(X, Y)$ such that $U(f)=\int_{T} f(t) d m(t)$, for every $f$ in $M_{t}(T, X)$.

Proof. Define the measure $m: H(T) \rightarrow L_{r}(X, Y)$ by the formula

$$
m(A)(x):=U\left(c_{A} x\right) \text { for all } A \in H(T) \text { and } x \in X .
$$

Now let $f \in M_{t}(T, X)$ and take an approximating net $\left(f_{\delta}\right)_{\delta \in \Delta}$ of $f$ in $S(T, X)$. Since $U\left(f_{\delta}\right)=\int_{T} f_{\delta}(t) d m(t)$ for every $\delta \in \Delta$ we get

$$
U(f)=o-\lim U\left(f_{\delta}\right)=o-\lim \int_{T} f_{\delta}(t) d m(t)=\int_{T} f(t) d m(t) .
$$

The uniqueness of $m$ is an evident fact.

\section{Similarly we can prove}

THEOREM 5.2. Let $U: \mathscr{L}^{1}(T, X) \rightarrow Y$ be a (po)-continuous positive linear operator. Then there exists a unique $\sigma$-measure $m: H_{\sigma}(T) \rightarrow L_{r}(X, Y)$ such that $U(f)=\int_{T} f(t) d m(t)$, for every $f$ in $\mathscr{L}^{1}(T, X)$.

Next let us denote by $\mathbf{O}\left(H(T), L_{r}(X, Y)\right)$ the p.o.v.s. of all differences of $L_{r}(X, Y)$-valued measures on the algebra $H(T)$.

The following assertion is an immediate consequence of Theorem 5.1.

THEOREM 5.3. $L_{r}\left(M_{t}(T, X), Y\right)_{u o}$ is isomorphic to $\mathbf{O}\left(H(T), L_{r}(X, Y)\right)$.

\section{Approximation and measurability in partially ordered normed spaces}

In this section, $X$ will denote a partially ordered normed space, $\|\cdot\|$ the corresponding norm, $\tau$ the norm (strong) topology of $X$ and $H_{\sigma}(X)$ the $\sigma$-algebra of Borel subsets of $X$.

An element $e>0$ of $X$ is called an orderunit norm if $\|x\| \leq k$ implies $-k \cdot e \leq x \leq k \cdot e, k \geq 0, x \in X$. 
The norm $\|\cdot\|$ is order continuous (respectively $\sigma$-continuous) on $X$ if $o$ - $\lim x_{\delta}=x\left(\right.$ respectively $o$ - $\lim x_{n}=x$ ) implies $o-\lim x_{\delta}=x$ (respectively 0 -lim $x_{n}=x$ ) whenever $\left(x_{\delta}\right)_{\delta \in \Delta}$ (respectively $\left(x_{n}\right)_{n \in \mathrm{N}}$ ) is a net (respectively sequence) in $X$ and $x \in X$.

The norm $\|\cdot\|$ is called absolute monotone on $X$ if $-y \leq x \leq y$ implies $\|x\| \leq\|y\|$, whenever $x, y \in X$.

Let $H_{\sigma}(T)$ be a $\sigma$-algebra of subsets of $T$ and $m: H_{\sigma}(T) \rightarrow Y$ a $\sigma$ measure. A function $f: T \rightarrow X$ is called $\left(H_{\sigma}(T), m\right)$-partitionable if for every neighborhood $G$ of 0 there is a countable partition $\left(A_{n}\right)_{n \in \mathbb{N}}$ of $T$ in $H_{\sigma}(T)$ such that $m\left(T-\bigcup_{n \in \mathbb{N}} A_{n}=0\right.$ and $f\left(A_{n}\right)-f\left(A_{n}\right) \subseteq G$, for all $n \in \mathbb{N}$.

Also $f$ is called totally bounded (respectively $\left(H_{\sigma}(T), H_{\sigma}(X)\right)$-measurable) if its range $f(T)$ is a totally bounded subset of $X$ (respectively $f^{-1}(F)$ $\in H_{\sigma}(T)$, whenever $\left.F \in H_{\sigma}(X)\right)$.

THEOREM 6.1. Let $f: T \rightarrow X$ be an $\left(H_{\sigma}(T), H_{\sigma}(X)\right)$-measurable function. Assume that $X$ is almost Archimedean, separable and possesses an orderunit norm $e$. Then $f \in M(T, X)$.

Proof. This can be proved analogously to [50, Theorem 4.1].

THEOREM 6.2. Let $f \in M(T, X)$. Assume that $X$ is almost Archimedean and the norm $\|\cdot\|$ is absolute monotone. Then

(i) $f$ is $\left(H_{\sigma}(T), m\right)$-partitionable,

(ii) $f$ is $\left(H_{\sigma}(T), H_{\sigma}(X)\right)$-measurable.

Proof. This is proved similarly to [50, Theorem 4.2].

Theorem 6.3. Let $f \in M_{t}(T, X)$. Suppose that the norm $\|\cdot\|$ of $X$ is absolute monotone and order continuous. Then $f$ is totally bounded.

Proof. There exists nets $\left(f_{\delta}\right)_{\delta \in \Delta}$ and $\left(x_{\delta}\right)_{\delta \in \Delta}$ in $S(T, X)$ and $X^{+}$, respectively, such that $-x_{\delta} \leq f_{\delta}(t)-f(t) \leq x_{\delta}$, for every $t \in T$ and $x_{\delta} \downarrow 0$. Hence the assertion follows from [40, page 70].

THEOREM 6.4. Let $f: T \rightarrow X$ be a totally bounded and $\left(H_{\sigma}(T), H_{\sigma}(X)\right)$ measurable function. Assume that $X$ is almost Archimedean and has an orderunit norm $e$. Then $f \in M_{t}(T, X)$.

Proof. Clearly, given $n \in \mathbb{N}$ there exist a finite partition $\left(A_{i}\right), i=$ $1,2, \ldots, k_{n}$ of $T$ and a sequence $\left(f_{n}:=\sum_{i=1}^{k_{n}} c_{A_{i}} a_{i}\right)_{n \in \mathbb{N}}$ in $S(T, X)$ such 
that $-\frac{1}{n} e \leq f_{n}(t)-f(t) \leq \frac{1}{n} e$, whenever $(n, t) \in \mathbb{N} \times T$. Consequently, the desired result follows.

We shall now turn our attention to the following cases. Let $T$ be a normal (respectively locally compact) topological space and $C_{t b}(T, X)$ (respectively $\left.C_{00}(T, X)\right)$ the partially ordered normed space of continuous totally bounded (respectively with compact support) functions from $T$ into $X$ endowed with pointwise ordering and uniform topology $\tau_{u}$. In particular we write $C_{t b}(T)$ (respectively $C_{00}(T)$ ) for $C_{t b}(T, R)$ (respectively $\left.C_{00}(T, R)\right)$.

THEOREM 6.5. Suppose that $X$ is almost Archimedean and has an orderunit norm $e$. Then

(i) for every $f$ in $C_{t b}(T, X)$ (respectively $C_{o o}(T, X)$ ) there exists $a$ sequence $\left(g_{n}:=\sum_{i=1}^{k_{n}} f_{i} x_{i}\right)_{n \in \mathbb{N}}$ with $f_{i} \in C_{t b}(T)$ (respectively $f_{i} \in C_{o o}(T)$ ), $x_{i} \in X, i=1,2, \ldots, k_{n}$, and $n \in \mathbb{N}$ such that $u$-lim $g_{n}=f$ on $T$.

(ii) $C_{t b}(T, X) \leqq M_{t}(T, X)$ (respectively $C_{o o}(T, X) \leqq M_{t}(T, X)$ ).

Proof. (i) Given $f \in C_{t b}(T, X)$ (respectively $f \in C_{o o}(T, X)$ ) and $n \in$ $\mathbb{N}$ there exist $x_{i} \in X, i=1,2, \ldots, k_{n}$, with

$$
T=\bigcup_{i=1}^{k_{n}} f^{-1}\left(B_{1 / n}\left(x_{i}\right)\right)
$$

where $B_{1 / n}\left(x_{i}\right)$ denotes the open sphere with center $x_{i}$ and radius $1 / n$.

Consider a continuous partition of unity $\left(f_{i}\right)$ subordinated to the preceding finite open covering of $T, i=1,2, \ldots, k_{n}$.

Therefore

$$
-\frac{1}{n} e \leq \sum_{i=1}^{k_{n}} f_{i}(t) x_{i}-f(t) \leq \frac{1}{n} e,
$$

whenever $t \in T$, which implies the desired result.

(ii) The assertions follow from Theorem 6.4.

Corollary 6.6. Suppose that $X$ is almost Archimedean and has an orderunit norm $e$. Then

(i) for every $f$ in $C_{t b}(T, X)^{+}$(respectively $C_{o o}(T, X)^{+}$) there exists a sequence $\left(g_{n}:=\sum_{i=1}^{k_{n}} f_{i} \cdot x_{i}\right)_{n \in \mathbf{N}}$ with $f_{i} \in C_{t b}(T)^{+}$(respectively $f_{i} \in$ $\left.C_{o o}(T)^{+}\right), x_{i} \in X^{+}, i=1,2, \ldots, k_{n}$, and $n \in \mathbb{N}$ such that

$$
u-\lim g_{n}=f \text { on } T \text {. }
$$

(ii) $C(T, X)^{+} \subseteq M_{t}(T, X)^{+}$(respectively $C_{o o}(T, X)^{+} \subseteq M_{t}(T, X)^{+}$). 


\section{Some classes of operators in partially ordered normed spaces}

Let $X$ be a partially ordered normed space and $Y$ a p.o.v.s. A linear oper-

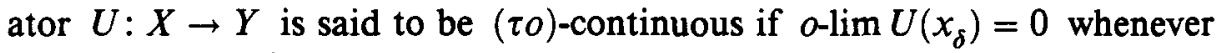
$\left(x_{\delta}\right)_{\delta \in \Delta}$ is a net in $X$ that $\tau$-converges to $0\left(\tau-\lim x_{\delta}=0\right)$.

We denote by $L_{r}(X, Y)_{\tau o}$ the p.o.v.s of all differences of positive $(\tau o)$ continuous linear operators from $X$ into $Y$. In particular we write $X_{\tau}^{\sim}$ for $L_{r}(X, R)_{\tau o}$.

As is easily verified, if $U \in L_{r}(X, Y)_{\tau o}$ and $X, Y$ are positively generated then $U^{\prime}$ (the restriction of $U^{\sim}$ on $Y_{\gamma}^{\sim}$ ) maps $Y_{\gamma}^{\sim}$ into $X_{\tau}^{\sim}$. Moreover $U^{\prime \prime}:\left(X_{\tau}^{\sim}\right)_{\gamma}^{\sim} \rightarrow\left(Y_{\gamma}^{\sim}\right)_{\gamma}^{\sim}$ is order continuous.

Throughout this section, assume that $T$ denotes a Hausdorff topological space and $Q(T, X)$ a p.o.v. subspace of $F(T, X)$ with $f(T)$ norm bounded for every $f$ in $Q(T, X)$, endowed with the uniform topology $\tau_{u}$ and the induced pointwise ordering from $F(T, X)$.

Now we proceed to discuss connections between $\left(\tau_{u} o\right)$ - and (uo)-continuous operators from $Q(T, X)$ into $Y$.

THEOREM 7.1. Let $U: Q(T, X) \rightarrow Y$ be a linear (uo)-continuous operator. Assume that $X$ is almost Archimedean and has an orderunit norm $e$. Then $U$ is $\left(\tau_{u} o\right)$-continuous.

Proof. Let $\left(f_{\delta}\right)_{\delta \in \Delta}$ be a net in $Q(T, X)$ with $\tau_{u}-\lim f_{\delta}=0$. Thus there exists a real decreasing net $\left(r_{\delta}\right)_{\delta \in \Delta}$ such that $\left\|f_{\delta}(t)\right\| \leq \sup \left\{\left\|f_{\delta}(t)\right\|: t \in T\right\} \leq$ $r_{\delta}$, for every $(\delta, t) \in \Delta \times T$ and $r_{\delta} \downarrow 0$. Hence $u$-lim $f_{\delta}=0$ on $T$, which implies $o-\lim U\left(f_{\delta}\right)=0$.

Using similar arguments we can prove the following

TheOREM 7.2. Let $U: Q(T, X) \rightarrow Y$ be a $\left(\tau_{u} o\right)$-continuous linear operator. Suppose that the norm $\|\cdot\|$ of $X$ is absolute monotone and order continuous. Then $U$ is (uo)-continuous.

\section{Alexandroff-type integral representation theorems}

In this section, we assume that $X$ is a positively generated almost Archimedean partially ordered normed space with an orderunit norm $e$ and $Y$ a $\gamma$-separative (respectively $\sigma$-separative) positively generated p.o.v.s.

We denote by $\phi\left(H(T), X_{\tau}^{\sim}\right)$ the p.o.v.s. of all differences of $X_{\tau}^{\sim}$-valued measures on the Borel algebra $H(T)$ of a normal topological space $T$ such 
that $\mu_{x}: H(T) \rightarrow R$ with $\mu_{x}(A):=\mu(A)(x)$ is a real-valued regular Borel measure, whenever $x \in X^{+}, A \in H(T)$ and $\mu \in \phi\left(H(T), X_{\tau}^{\sim}\right)^{+}$.

We also denote by

$$
\psi\left(H(T), L_{r}\left(X , ( Y _ { \gamma } ^ { \sim } { \tilde { \gamma _ { \tau _ { 0 } } } } ^ { \sim } ) \text { (respectively } \psi \left(H(T), L_{r}\left(X,\left(Y_{\sigma}^{\sim}\right)_{\gamma_{\tau o}}^{\sim}\right)\right.\right.\right.
$$

the p.o.v.s. of all differences of

$$
L_{r}\left(X,\left(Y_{\gamma}^{\sim}\right)_{\gamma}^{\sim}\right)_{\tau 0^{-}} \quad\left(\text { respectively } L_{r}\left(X,\left(Y_{\sigma}^{\sim}\right)_{\gamma}^{\sim}\right)_{\tau 0^{-}}\right)
$$

valued measures on the Borel algebra $H(T)$ such that

$$
m_{y^{\sim}} \in \phi\left(H(T), X_{\tau}^{\sim}\right) \text { with } m_{y^{\sim}}(A):=m(A)(x)\left(y^{\sim}\right),
$$

whenever $x \in X, y^{\sim} \in Y_{\gamma}^{\sim}$ (respectively $\left.y^{\sim} \in Y_{\sigma}^{\sim}\right) y^{\sim} \geq 0$ and

$$
m \in \psi\left(H(T), L_{r}\left(X,\left(Y_{\gamma}^{\sim}\right)_{\gamma_{\tau o}}^{\sim}\right) \quad\left(\text { respectively } m \in \psi\left(H(T),\left(Y_{\sigma}^{\sim}\right)_{\gamma_{\tau o}}^{\sim}\right)\right. \text { ). }\right.
$$

THEOREM 8.1. Let $w$ be a positive element of $C_{t b}(T, X)_{\tau_{u}}^{\sim}$. Then there exists exactly one Borel measure $\mu$ in $\phi\left(H(T), X_{\tau}^{\sim}\right)$ such that $w(f)=$ $\int_{T} f(t) d \mu(t)$, for every $f$ in $C_{t b}(T, X)$.

Proof. Let $x \in X^{+}$. Define $C_{t b}^{x}(T, X):=\left\{h \cdot x: h \in C_{t b}(T)\right\}$. Clearly $C_{t b}^{x}(T, X)$ is algebraic and order isomorphic to $C_{t b}(T)$. Therefore we identify $C_{t b}^{x}(T, X)$ with $C_{t b}(T)$.

Thus by the scalar Alexandroff representation theorem [4, page 577] there exists a unique regular real-valued measure $\mu_{x}: H(T) \rightarrow R$ such that

$$
w_{x}(h)=w(h \cdot x)=\int_{T} h(t) d \mu_{x}(t), \quad \text { for every } h \in C_{t b}(T),
$$

where $w_{x}$ is the restriction of $w$ to $C_{t b}(T)$.

Extend (1) to be true for arbitrary $x \in X$ with $x=x_{1}-x_{2}, x_{i} \geq 0$, $i=1,2$, by the formula

$$
w_{x}(h)=w_{x_{1}-x_{2}}(h):=\int_{T} h(t) d \mu_{x_{1}}(t)-\int_{T} h(t) d \mu_{x_{2}}(t),
$$

for every $h \in C_{t b}(T)$. Clearly $w_{x} \in C_{t b}(T)_{\tau_{u}}^{\sim}$.

Next define a measure $\mu: H(T) \rightarrow X_{\tau}^{\sim}$ by the relation

$$
\mu(A)(x):=\mu_{x}(A), \quad A \in H(T), x \in X .
$$

On the other hand, for each $h \in C_{t b}(T)$ and $x \in X$, we have

$$
w(h \cdot x)=\int_{T} h(t) x d \mu(t)=\int_{T} h(t) d \mu_{x}(t),
$$

obtained by using an approximating sequence in $S(T, R)$ of $h \in C_{t b}(T)$ and by applying Theorem 4.1 (ii).

Moreover Theorem 6.5 guarantees that $\mu$ represents $w$ on the whole space $C_{t b}(T, X)$. Its uniqueness is evident. 
THEOREM 8.2. Let $U$ be a positive element in $L_{r}\left(C_{t b}(T, X), Y\right)_{\tau_{u} o}$. Then there exists exactly one measure $m \in \psi\left(H(T), L_{r}\left(X,\left(Y_{\gamma}^{\sim}\right)_{\gamma}^{\sim}\right)_{\tau o}\right.$ ) (respectively $m \in \psi\left(H(T), L_{r}\left(X,\left(Y_{\sigma}^{\sim}\right)_{\gamma}^{\sim}\right)_{\tau o}\right)$ such that $U(f)=\int_{T} f(t) d m(t)$, for every $f$ in $C_{t b}(T, X)$.

Proof. Let $y^{\sim} \in Y_{y}^{\sim}$ (respectively $y^{\sim} \in Y_{\sigma}^{\sim}$ ) with $y^{\sim} \geq 0$. Consider the positive linear $\tau_{u}$-continuous functional $U_{y^{\sim}}: C_{t b}(T, X) \rightarrow R$, defined by the formula $U_{y^{\sim}}(f)=y^{\sim}(U(f))$, for every $f \in C_{t b}(T, X)$.

Theorem 8.1 guarantees the existence of exactly one measure $m_{y}$ in $\varphi\left(H(T), X_{\tau}^{\sim}\right)$ such that $U_{y^{\sim}}(f)=\int_{T} f(t) d m_{y^{\sim}}(t)$, for all $f$ in $C_{t b}(T, X)$.

Now we define a measure $m: H(T) \rightarrow L_{r}\left(X,\left(Y_{\gamma}^{\sim}\right)_{\gamma}^{\sim}\right)_{\tau o}$ (respectively $m$ : $\left.H(T) \rightarrow L_{r}\left(X,\left(Y_{\sigma}^{\sim}\right)_{\gamma}^{\sim}\right)_{\tau o}\right)$ by the relation

$$
m(A)(x)\left(y^{\sim}\right):=m_{y^{\sim}}(A)(x)
$$

whenever $A \in H(T), x \in X$, and the corresponding integral operator

$$
U_{1}: M_{t}(T, X) \rightarrow\left(Y_{\gamma}^{\sim}\right)_{\gamma}^{\sim} \quad\left(\text { respectively } U_{1}: M_{t}(T, X) \rightarrow\left(Y_{\gamma}^{\sim}\right)_{\gamma}^{\sim}\right)
$$

by the relation $U_{1}(f):=\int_{T} f(t) d m(t)$, whenever $f \in M_{t}(T, X)$.

Since $C_{t b}(T, X) \subseteq M_{t}(T, X)$ we easily establish that

$$
U_{1}(f)=U(f)=\int_{T} f(t) d m(t)
$$

for every $f$ in $C_{t b}(T, X)$.

To prove uniqueness let $l$ be another measure in

$$
\psi\left(H(T), L_{r}\left(X,\left(Y_{\gamma}^{\sim}\right)_{\gamma}^{\sim}\right)_{\tau o}\right) \quad\left(\text { respectively } \psi\left(H(T), L_{r}\left(X,\left(Y_{\sigma}^{\sim}\right)_{\gamma}^{\sim}\right)_{\tau o}\right)\right)
$$

such that

$$
U(f)=\int_{T} f(t) d m(t)=\int_{T} f(t) d l(t)
$$

whenever $f \in C_{t b}(T, X)$. Consequently $\int_{T} f(t) d m_{y^{\sim}}(t)=\int_{T} f(t) d l_{y^{\sim}}(t)$, for every $f \in C_{t b}(T, X)$, which implies $m=l$.

THEOREM 8.3. $C_{t b}(T, X)_{\tau_{u}}^{\sim}$ is isomorphic to $\varphi\left(H(T), X_{\tau}^{\sim}\right)$.

Proof. By the integration process, each $\mu$ in $\phi\left(H(T), X_{\tau}^{\sim}\right)$ defines a positive functional $w$ in $C_{t b}(T, X)_{\tau_{u}}^{\sim}$ and the correspondence $\xi: \phi\left(H(T), X_{\tau}^{\sim}\right)$ $\rightarrow C_{t b}(T, X)_{\tau_{u}}^{\sim}$ defined by $\xi(\mu):=w$ is positive and linear.

Again, from the scalar Alexandroff representation theorem it is an easy matter to prove that $\xi$ is one-to-one.

On the other hand Theorem 8.1 guarantees that $\xi$ is onto. This completes the proof of the assertion. 
THEOREM 8.4. Suppose that $Y$ is order reflexive. Then $L_{r}\left(C_{t b}(T, X), Y\right)_{\tau_{u} o}$ is isomorphic to $\psi\left(H(T), L_{r}(X, Y)_{\tau o}\right)$.

Proof. This is proved analogously to Theorem 8.3.

\section{Riesz-type integral representation theorems}

Let $X$ be a partially ordered normed space and $Y$ a p.o.v.s. provided with the properties of the foregoing section.

We denote by $\phi_{\sigma}\left(H_{\sigma}(T), X_{\tau}^{\sim}\right)$ the p.o.v.s. of all differences of $X_{\tau}^{\sim}$-valued $\sigma$-measures on the Borel $\sigma$-algebras $H_{\sigma}(T)$ of a locally compact topological space $T$ such that $\mu_{x}: H_{\sigma}(T) \rightarrow R$ with $\mu_{x}(A):=\mu(A)(x)$ is a realvalued regular Borel measure, whenever $x \in X^{+}, A \in H_{\sigma}(T)$ and $\mu \in$ $\phi_{\sigma}\left(H_{\sigma}(T), X_{\tau}^{\sim}\right)^{+}$.

We also denote by

$$
\psi_{\sigma}\left(H_{\sigma}(T), L_{r}\left(X,\left(Y_{\gamma}^{\sim}\right)_{\gamma}^{\sim}\right)_{\tau o}\right) \quad\left(\text { respectively } \psi_{\sigma}\left(H_{\sigma}(T), L_{r}\left(X,\left(Y_{\sigma}^{\sim}\right)_{\gamma}^{\sim}\right)_{\tau o}\right)\right)
$$

the p.o.v.s. of all differences of

$$
L_{r}\left(X,\left(Y_{\gamma}^{\sim}\right)_{\gamma}^{\sim}\right)_{\tau o^{-}} \quad\left(\text { respectively } L_{r}\left(X,\left(Y_{\sigma}^{\sim}\right)_{\gamma}^{\sim}\right)_{\tau o^{-}}\right)
$$

valued $\sigma$-measures on the Borel $\sigma$-algebra $H_{\sigma}(T)$ such that

$$
m_{y^{\sim}} \in \phi_{\sigma}\left(H_{\sigma}(T), X_{\tau}^{\sim}\right) \text { with } m_{y^{\sim}}(A):=m(A)(x)\left(y^{\sim}\right),
$$

whenever $x \in X, y^{\sim} \in Y_{\gamma}^{\sim}$ (respectively $\left.y^{\sim} \in Y_{\sigma}^{\sim}\right), y^{\sim} \geq 0$ and $m \in \psi_{\sigma}\left(H_{\sigma}(T), L_{r}\left(X,\left(Y_{\gamma}^{\sim}\right)_{\gamma}^{\sim}\right)_{\tau o}\right)$ (respectively $\left.m \in \psi_{\sigma}\left(H_{\sigma}(T),\left(Y_{\sigma}^{\sim}\right)_{\gamma}^{\sim}\right)_{\tau o}\right)$.

Now we can easily obtain proofs of the following theorems by using similar arguments to those of the corresponding theorems of the preceding section.

THEOREM 9.1. Let $w$ be a positive element of $C_{o o}(T, X)_{\tau_{u}}^{\sim}$. Then there exists exactly one Borel $\sigma$-measure in $\phi_{\sigma}\left(H_{\sigma}(T), X_{\tau}^{\sim}\right)$ such that $w(f)=$ $\int_{T} f(t) d \mu(t)$, for every $f$ in $C_{o o}(T, X)$.

THEOREM 9.2. Let $U$ be a positive element in $L_{r}\left(C_{o o}(T, X), Y\right)_{\tau_{u} o}$. Then there exists exactly one $\sigma$-measure $m \in \psi_{\sigma}\left(H_{\sigma}(T), L_{r}\left(X,\left(Y_{\gamma}^{\sim}\right)_{\gamma}^{\sim}\right)_{\tau o}\right)^{u^{o}}$ (respectively $\left.m \in \psi_{\sigma}\left(H_{\sigma}(T), L_{r}\left(X,\left(Y_{\sigma}^{\sim}\right)_{\gamma}^{\sim}\right)_{\tau o}\right)\right)$ such that $U(f)=\int_{T} f(t) d m(t)$, for every $f$ in $C_{o o}(T, X)$.

THEOREM 9.3. $C_{o o}(T, X)_{\tau_{u}}^{\sim}$ is isomorphic to $\phi_{\sigma}\left(H_{\sigma}(T), X_{\tau}^{\sim}\right)$. 
Theorem 9.4. Suppose that $Y$ is order reflexive. Then

$$
L_{r}\left(C_{o o}(T, X), Y\right)_{\tau_{u} o}
$$

is isomorphic to $\psi_{\sigma}\left(H_{\sigma}(T), L_{r}(X, Y)_{\tau o}\right)$.

\section{Multiplicative operators}

We start with the following assumptions. Let $X$ be a partially ordered normed algebra positively generated by a normal cone $X^{+}$with a separately order continuous multiplication, $T$ a normal topological space and $H(T)$ its Borel algebra.

Then $C_{t b}(T, X)$ is also a partially ordered normed algebra positively generated by a normal cone under the usual uniform topology $\tau_{u}$ and pointwise algebraic-order structure.

As is also well known, $C_{t b}(T, X)_{\tau_{u}}^{\sim}=C_{t b}(T, X)^{\prime}$ (the topological dual of $\left.C_{t b}(T, X)\right)$. Therefore $C_{t b}(T, X)_{\tau_{u}}^{\sim}$ is a separating p.o.v. subspace of $C_{t b}(T, X)^{\sim}$.

Let $Y$ be another partially ordered algebra. Assume that $Y$ is monotone complete $\gamma$ - (respectively $\sigma-$ ) separative and positively generated.

A positive operator $U$ in $L_{r}\left(C_{t b}(T, X), Y\right)$ is called multiplicative if $U(f \cdot g)=U(f) \cdot U(g)$, whenever $f, g \in C_{t b}(T, X)$.

We continue with a useful criterion for multiplicativity.

LemMa 10.1. Let $U$ be a positive element in $L_{r}\left(C_{t b}(T, X), Y\right)_{\tau_{u} o}$. Then $U$ is multiplicative if and only if $U^{\prime \prime}(A \cdot B)=U^{\prime \prime}(A) \cdot U^{\prime \prime}(B)$, for all $A, B$ in $\left(C_{t b}(T, X)_{\tau_{u}}^{\sim}\right)_{\gamma}^{\sim}$.

Proof. In order to prove the sufficiency, take any $A, B$ in $\left(C_{t b}(T, X)_{\tau_{u}}^{\sim}\right)_{\gamma}^{\sim}$ and $y^{\sim} \in Y_{\gamma}^{\sim}$ (respectively $\left.y^{\sim} \in Y_{\sigma}^{\sim}\right), y^{\sim} \geq 0$. Thus $U^{\prime \prime}(A \cdot B)\left(y^{\sim}\right)=$ $(A \cdot B)\left(U^{\prime}\left(y^{\sim}\right)\right)=A \cdot\left(B \cdot U^{\prime}\left(y^{\sim}\right)\right),\left(U^{\prime \prime}(A) U^{\prime \prime}(B)\right)\left(y^{\sim}\right)=A \cdot U^{\prime}\left(U^{\prime \prime}(B)\left(y^{\sim}\right)\right)$. Also if $f \in C_{t b}(T, X)$ we have

$$
\left(B \cdot U^{\prime}\left(y^{\sim}\right)\right)(f)=B \cdot\left(U^{\prime}\left(y^{\sim}\right)\right)_{f} \text { and } U^{\prime}\left(U^{\prime \prime}(B)\left(y^{\sim}\right)(f)=B\left(U^{\prime}\left(y_{U(f)}^{\sim}\right)\right)\right. \text {. }
$$

Furthermore for $g \in C_{t b}(T, X)$ we deduce that

$$
U^{\prime}\left(y^{\sim}\right)_{f}(g)=y^{\sim}(U(f \cdot g)) \text { and } U^{\prime}\left(y_{U(f)}^{\sim}\right)(g)=y^{\sim}(U(f \cdot g)) \text {. }
$$

Hence $U^{\prime \prime}(A \cdot B)=U^{\prime \prime}(A) \cdot U^{\prime \prime}(B)$. 
Necessity follows from $C_{t b}(T, X) \leqq\left(C_{t b}(T, X)_{\tau_{u}}^{\sim}\right)_{\gamma}^{\sim}$.

On the other hand, in view of preceding definitions and results, the following lemmas can be easily established.

Lemma 10.2. Let $A \in H(T)$ and $x \in X$. Then the function $\left[c_{A} x\right]$ : $C_{t b}(T, X)_{\tau_{u}}^{\sim}=\phi\left(H(T), X_{\tau}^{\sim}\right) \rightarrow R$ defined by the formula

$$
\left[c_{A} x\right](m):=\int_{T} c_{A}(t) x d m(t),
$$

whenever $m \in \phi\left(H(T), X_{\tau}^{\sim}\right)$, belongs in $\left(C_{t b}(T, X)_{\tau_{u}}^{\sim}\right)_{\gamma}^{\sim}$.

LEMMA 10.3. The representing measure $m \in \psi\left(H(T), L_{r}\left(X,\left(Y_{\gamma}^{\sim}\right)_{\gamma}^{\sim}\right)_{\tau o}\right)$ (respectively $m \in \psi\left(H(T), L_{r}\left(X,\left(Y_{\sigma}^{\sim}\right)_{\gamma}^{\sim}\right)_{\tau o}\right)$ ) of a positive operator $U \in$ $L_{r}\left(C_{t b}(T, X), Y\right)_{\tau_{u} o}$ is given by the formula $m(A) x=U^{\prime \prime}\left(\left[c_{A} x\right]\right)$, whenever $A \in H(T)$ and $x \in X$.

LEMMA 10.4. $\left(\left[c_{A_{1}} x_{1}\right]\right) \cdot\left(\left[c_{A_{2}} x_{2}\right]\right)=\left[c_{A_{1} \cap A_{2}}\left(x_{1} \cdot x_{2}\right)\right]$, for all $A_{1}, A_{2}$ in $H(T)$ and $x_{1}, x_{2}$ in $X$.

Now we state and prove the main results of this section.

THEOREM 10.5. A positive element $U$ in $L_{r}\left(C_{t b}(T, X), Y\right)_{\tau_{u} o}$ is multiplicative if and only if the representing measure $m$ in

$$
\psi\left(H(T), L_{r}\left(X,\left(Y_{\gamma}^{\sim}\right)_{\gamma}^{\sim}\right)_{\tau o}\right)
$$

(respectively $\left.\psi\left(H(T), L_{r}\left(X,\left(Y_{\sigma}^{\sim}\right)_{\gamma}^{\sim}\right)_{\tau o}\right)\right)$ satisfies

$$
m\left(A_{1} \cap A_{2}\right)\left(x_{1} \cdot x_{2}\right)=\left(m\left(A_{1}\right)\left(x_{1}\right)\right) \cdot\left(m\left(A_{2}\right)\left(x_{2}\right)\right),
$$

for all $x_{1}, x_{2}$ in $X$ and $A_{1}, A_{2}$ in $H(T)$.

Proof. First suppose that $U$ is multiplicative. Then it follows easily by Lemmas 10.2 and 10.3 that

$$
m\left(A_{1} \cap A_{2}\right)\left(x_{1} \cdot x_{2}\right)=\left(m\left(A_{1}\right)\left(x_{1}\right)\right) \cdot\left(m\left(A_{2}\right)\left(x_{2}\right)\right),
$$

whenever $x_{1}, x_{2} \in X$ and $A_{1}, A_{2} \in H(T)$.

Conversely suppose that the preceding condition holds. Then for arbitrary $f, g$ in $C_{t b}(T, X)$, a similar argument to that in the last part of the proof 
of [15, Theorem 22] indicates that

$$
\int_{T} f(t) g(t) d m(t)=\int_{T} f(t) d m(t) \cdot \int_{T} g(t) d m(t) .
$$

This completes the proof.

When $T$ is a locally compact topological space and $H_{\sigma}(T)$ the Borel $\sigma$ algebra of subsets of $T$, using similar arguments, we can prove the following.

THEOREM 10.6. A positive element $U$ in $L_{r}\left(C_{o o}(T, X), Y\right)_{\tau_{u} o}$ is multiplicative if and only if the representing $\sigma$-measure $m$ in

$$
\psi_{\sigma}\left(H_{\sigma}(T), L_{r}\left(X,\left(Y_{\gamma}^{\sim}\right)_{\gamma}^{\sim}\right)_{\tau o}\right)
$$

(respectively $\psi_{\sigma}\left(H_{\sigma}(T), L_{r}\left(X,\left(Y_{\sigma}^{\sim}\right)_{\gamma}^{\sim}\right)_{\tau o}\right)$ ) satisfies

$$
m\left(A_{1} \cap A_{2}\right)\left(x_{1} \cdot x_{2}\right)=\left(m\left(A_{1}\right)\left(x_{1}\right)\right) \cdot\left(m\left(A_{2}\right)\left(x_{2}\right)\right),
$$

for all $x_{1}, x_{2}$ in $X$ and $A_{1}, A_{2}$ in $H_{\sigma}(T)$.

\section{Applications}

Our results found significant and important applications in partially ordered vector spaces of operators acting usually on a Hilbert space $H$. We shall briefly outline some definitions and principal results concerning such kinds of operator spaces.

For more details in this direction consult $[2,3,10,18,23-24,29,30,32$, $55,59,61,65-67,70,74,80-82]$.

(i) $C^{*}$-algebras. Let $\mathscr{A}$ be a $C^{*}$-algebra with identity $e, H$ its universal representation space, $A$ the self-adjoint part of $\mathscr{A}$ and $L(H, H)$ the bounded linear operators acting on $H$.

We recall that $A$ is a partially ordered Banach space with positive cone $\left\{a^{*} \cdot a: a \in \mathscr{A}\right\}$ and $\mathscr{A}$ may be regarded as a subalgebra of $L(H, H)$. Also $A$ is an orderunit space with orderunit and orderunit norm $e$; moreover the orderunit norm on $A$ coincides with the $C^{*}$-norm.

We call $\mathscr{A}$ monotone (respectively $\sigma$-monotone) complete if $A$ is monotone (respectively $\sigma$-monotone) complete. Each $W^{*}$-algebra and each $A W^{*}$ algebra of type $I$ is monotone complete.

Let $A_{s}$ be a subalgebra of $\mathscr{A}$ of self-adjoint elements.

Our presented results can be immediately applied in the following cases:

$$
X=A, \quad Y=A^{\prime}, \quad Z=R ; \quad \text { or } \quad X=Y=Z=A_{s} \text {. }
$$

(ii) Jordan algebras. Let $J$ be a Jordan algebra of selfadjoint operators acting on $H$, that is, a real vector space of such operators closed under the product $U \odot V:=\frac{1}{2}(U \cdot V+V \cdot U)$. 
By a Banach Jordan algebra $J$ we mean a real Banach space and a Jordan algebra such that $\|U \odot V\| \leq\|U\| \cdot\|V\|$, whenever $U, V \in J$. It is called a $J B$-algebra if moreover $J$ has a unit $\left\|U^{2}\right\|=\|U\|^{2}$ and $\left\|U^{2}\right\| \leq\left\|U^{2}+V^{2}\right\|$, for all $U, V \in J$.

A $J C$-algebra is a uniformly closed Jordan algebra with unit.

Alfsen, Shultz and Størmer proved in[2] that if $J$ is a $J B$-algebra with identity $I$ then the set $\left\{U^{2}: U \in J\right\}$ is a (proper) closed convex cone under which $J$ becomes a partially ordered Banach space with $I$ as an orderunit norm and $-I \leq U \leq I$ implies $0 \leq U^{2} \leq I$, whenever $U \in J$.

It is well known that every $J C$-algebra is a $J B$-algebra but the converse is false.

Our included results are directly applied to the cases:

$$
X=J, \quad Y=J^{\prime}, \quad Z=R ; \quad \text { and } X=Y=Z=J .
$$

(iii) Semifields and $O^{*}$-algebras. An associative, commutative partially ordered algebra $E$ is called a semifield if it satisfies the following conditions.

$\left(s_{1}\right) E$ contains a subset $K$ such that $K+\bar{K} \subseteq K$ and $K \cdot K \subseteq K$ with $\bar{K}:=\left\{x \in E:\right.$ there exists a sequence $\left(x_{n}\right)_{n \in \mathbb{N}}$ in $K$ such that $\left.x=0-\lim x_{n}\right\}$.

$\left(s_{2}\right) \quad E=K-K$

$\left(s_{3}\right)$ There exists $\sup \left\{x_{n}: n \in \mathbb{N}\right\}$ in $E$ for every bounded sequence $\left(x_{n}\right)_{n \in \mathbb{N}}$ in $E$.

$\left(s_{3}\right) E$ has more than one element and the equation $a \cdot x=b$ has at least one solution which is an element of $K$ whenever $a, b \in K$.

$\left(s_{5}\right) \bar{K}$ coincides with the set of all non-negative elements of $E$.

We note that the class of semifields defined here is much wider than the class of topological semifields studied in [3] and is a subclass of $K_{\sigma}$-spaces developed and investigated by L. V. Kantorovich, B. Z. Vulikh and A. G. Pinsker in [37, 38, 74].

Now a $*$-involutory (complex) algebra $\mathscr{A}$ with unit $e$ is called an $O^{*}$ algebra if there is defined a partial ordering on the vector space $A$ of selfadjoint elements of $\mathscr{A}$, compatible with the algebraic operations and satisfying the following axioms:

$\left(o_{1}\right) A^{+}+A^{+} \subseteq A^{+}:=\{a \in A: a \geq 0\}$ and $\left(a^{*} \cdot a\right) \in A^{+}$for every $a \in A$;

$\left(o_{2}\right)$ for every majorised increasing net $\left(a_{\delta}\right)_{\delta \in \Delta}$ in $A$ there exists $a:=$ $\sup \left\{a_{\delta}: \delta \in \Delta\right\}$ in $A$ such that $a \cdot b=b \cdot a$ provided $a_{\delta} \cdot b=b \cdot a_{\delta}$, whenever $\delta \in \Delta$ and $b \in A$;

$\left(o_{3}\right)$ every maximal commutative $*$-subalgebra of $\mathscr{A}$ is a (complex) semifield.

We provided important applications of our results in the following cases: $X=E, Y=E^{\sim}, Z=R$ or $X=Y=Z=E$, with $E$ a semifield and $X=A^{+}-A^{+}, Y=A^{\prime}, Z=R$ or $X=Y=Z=A_{s}$, whenever $A$ is the 
self-adjoint part of an $O^{*}$-algebra $\mathscr{A}$ and $A_{s}$ an algebra of elements of $A$.

(iv) Quantum probability theory. E. B. Davies and J. T. Lewis, in order to describe repeated measurements in quantum mechanical systems, have defined in [20] the concrete concept of quantum instrument.

More precisely, let $\left(X, X^{+}, e\right)$ be a triple consisting of a real Banach space $X$, (according to the terminology of [20] a state space), positively generated by the (strong) closed cone $X^{+}$and a positive linear functional $e: X \rightarrow R$ such that $e(x)=\|x\|$, whenever $x \in X^{+}$.

Assume moreover that $\|x\|=\inf \left\{\left\|x_{1}\right\|+\left\|x_{2}\right\|: x=x_{1}-x_{2}, x_{i} \in X^{+}, i=\right.$ $1,2\}$ for every $x \in X$.

As is easily verified, $X$ is monotone complete, the cone $X^{+}$is normal and $e \in X_{\gamma}^{\sim}$. Also every positive operator in $L_{r}(X, X)$ is continuous, and hence $L_{r}(X, X)=L_{\gamma}(X, X)$.

Let $H_{\sigma}(T)$ be a $\sigma$-algebra of subsets of a space $T$. In the terminology of [20] an observable is a positive set function $a: H_{\sigma}(T) \rightarrow X^{\prime}$ satisfying

$(o b)_{1} \quad 0 \leq a(A) \leq a(T)$, for all $A \in H_{\sigma}(T)$ and $a(T)=e$,

$(o b)_{2} \quad a$ is $\sigma$-additive with respect to the weak *-topology of $X^{\prime}$. We say $a$ is multiplicative if $a\left(A_{1} \cap A_{2}\right)=a\left(A_{1}\right) \cdot a\left(A_{2}\right)$, whenever $A_{1}, A_{2} \in H_{\sigma}(T)$. On the other hand an instrument is a positive set function $\mathscr{E}: H_{\sigma}(T) \rightarrow$ $L_{r}(X, X)$ such that

(in) $" e(\mathscr{E}(T)(x)=e(x)$, for every $x \in X$,

(in) ${ }_{2} " \mathscr{E}$ is $\sigma$-additive with respect to strong operator topology.

It is an easy matter to prove that $\mathscr{E}$ (respectively $a$ ) is an $L_{r}(X, X)$ $\sigma$-measure (respectively $X^{\prime}$-measure) on $H_{\sigma}(T)$. Furthermore, it has been shown in [20] that given an instrument $\mathscr{E}$ there is a unique observable $a$ such that $e(\mathscr{E}(A)(x))=a(A)(x)$, for all $x \in X$ and $A \in H_{\sigma}(T)$. Also every observable $a$ is determined in such a way by at least one instrument $\mathscr{E}$.

Recall that the example most significant to quantum mechanics is to take $X$ the self-adjoint trace class operators $\mathscr{T}_{s}(H)$ on a separable Hilbert space $H, e$ the usual trace on $X, T$ a separable locally compact topological space and $H_{\sigma}(T)$ the Borel $\sigma$-algebra of $T$.

As we may know, a corresponding integration process of $X-, X^{\prime}$ - or $L_{r}(X, X)$-valued random variables in a similar sense of E. Rowecka [54] and $H$. Umegaki and $A$. T. Bharucha-Reid [73] with respect to an $L_{r}(X, X)$ (respectively $X$-) instrument (respectively observable) is lacking from this operational approach to quantum probability theory.

So our presented results can be immediately applied to cover this missing process.

(v) The operator Feynman-Kac formula. Let $X$ be a partially ordered Banach space, $L(X, X)$ the space of bounded linear operators acting on $X$, 
$T$ a locally compact Hausdorff space, $H_{\sigma}(T)$ the $\sigma$-algebra of Borel or Baire subsets of $T$ and a $\sigma$-measure $m: H_{\sigma}(T) \rightarrow L(X, X)$.

Let $f: T \rightarrow R$ be a Borel or Baire function. Define the operator $m(f): X$ $\rightarrow X$ with $m(f)(x):=\int_{T} f(t) d m(t)(x)$, for every $x \in X$, whenever the right-hand side exists.

Given such a function $f$, we assume that the operator $m(f)$ is the infinitesimal generator of a $C_{0}$-semigroup $Q(u), u \in R^{+}$of linear operators on $X$ (cf. $[34,69]$ ).

We also consider another $C_{0}$-semigroup $W(u), u \in R^{+}$, of linear operators on $X$, whose infinitesimal generator is the operator $A$.

Now we are looking for a third semigroup $U(u), u \in R^{+}$, satisfying the initial value problem

$$
U^{\prime}(s)=A U(s)+m(f) U(s), \quad s>0, \quad U(0)=I .
$$

In many cases there are difficulties in deriving the semigroup $U(u), u \in$ $R^{+}$, since the preceding initial-value problem $(11.1)$ does not in general have classical or mild solutions.

So we try to construct $U(u), u \in R^{+}$, using an operator formula of the Feynman-Kac type. For this purpose let $s \in R^{+}$and let $T_{s}$ be the set of all continuous paths $p:[0, s] \rightarrow T$ based on the closed interval $[0, s]$. Let also $P_{s}$ be the semialgebra of sets

$$
A:=\left\{p \in T_{s}: p\left(s_{j}\right) \in B_{j}, j=1,2, \ldots, k\right\}
$$

for arbitrary $k=1,2, \ldots$,

$$
0 \leq s_{1}<s_{2}<\cdots<s_{k-1}<s_{k} \leq s \quad \text { and } B_{j} \in H_{\sigma}(T), \quad j=1,2, \ldots, k \text {. }
$$

Then a measure (respectively $\sigma$-measure) $m_{s}: H_{\sigma}\left(T_{s}\right) \rightarrow L(X, X)$ such that

$$
m_{s}(A):=W\left(s-s_{k}\right) m\left(B_{k}\right) W\left(s_{k}-s_{k-1}\right) m\left(B_{k-1}\right) \cdots W\left(s_{2}-s_{1}\right) m\left(B_{1}\right) W\left(s_{1}\right),
$$

for every $A \in P_{s}$, will be called a $(W, m, s)$-measure, where $H_{\sigma}\left(T_{s}\right)$ denotes the $\sigma$-algebra generated by $P_{s}$.

Now the semigroup $U(u), u \in R^{+}$, can be interpreted in the following operator formula of the Feynman-Kac type (cf. [42, 43]):

$$
U(s)=\int_{T_{s}}\left[\exp \left(\int_{0}^{s} f(p(r)) d r\right)\right] d m_{s}(p) .
$$

We recall that a Riemann-Stieltjes or Bochner-type integration process of R. G. Bartle, N. Dunford, J. Schwartz [5], R. G. Bartle [6], J. Batt and E. J. Berg [7], J. K. Brooks and P. W. Lewis [12], J. K. Brooks and N. Dinculeanu [13], N. Dinculeanu [22], I. Dobrakov [25, 26], N. Dunford [27], R. J. Easton and D. H. Tucker [28], J. R. Edwards and S. G. Wayment [31], 
R. K. Goodrich [34], A. Shuchat [64], K. Swong [68] and U. J. Uherka [72], cannot be applied to define the right hand side of (11.2) since $m_{s}$ is not in general of finite variation (cf. [43]).

On the other hand, our presented integration theory does not presuppose finite variation of the corresponding measures (respectively $\sigma$-measures) so the obtained integrals can be immediately applied to define the desired semigroup $U(u), u \in R^{+}$, from formula (11.2).

REMARK. In mathematical physics, the $C_{o}$-semigroup $W(u), u \in R^{+}$, usually describes the motion of a free quantum mechanical particle with $n$ degrees of freedom $\left(T=\mathbb{R}^{n}, n \geq 1\right)$ in a force field with a given potential, and the "solutions" $u(s)=U(s) x_{0}$ of (11.1) characterize the states of such a particle at time $s>0$, with initial state $x_{0}$ in $X=\mathscr{L}^{2}\left(\mathbb{R}^{n}\right)$ and superposition of $Q(u), W(u)$ the $C_{o}$-semigroup $U(u), u \in R^{+}$.

\section{References}

[1] E. M. Alfsen, Compact convex sets and boundary integrals, Springer-Verlag, 1971.

[2] E. M. Alfsen, F. W. Shultz and E. Størmer, 'A Gelfand-Neumark theorem for Jordan algebras, Adv. in Math. 28 (1978), 11-56.

[3] N. Ja. Antonovskii, V. G. Boltjanskii and T. A. Sarymsakov, Topological Boolean algebra, Izdat. Akad. Nauk Uzbek. SSR, Tashkent, 1963 (Russian). English translation, 'Topological semifields and their application to general topology', Amer. Math. Soc. Transl. 106 (1977).

[4] A. D. Alexandroff, 'Additive set functions in abstract spaces', Rec. Math. (Mat. Sbornik) N.S. (2) 51 (1941), 563-621.

[5] R. G. Bartle, N. Dunford and J. Schwartz, 'Weak compactness and vector measures', Canad. J. Math. 7 (1955), 289-305.

[6] R. G. Bartle, 'A general bilinear vector integral', Studia Math. 15 (1956), 337-352.

[7] J. Batt and E. J. Berg, 'Linear bounded transformations on the space of continuous functions', J. Funct. Anal. 4 (1969), 215-239.

[8] S. K. Berberian, Notes on spectral theory, Van Nostrand, Princeton, N.J., 1966.

[9] S. Bochner, 'Integration and differentiation in partially ordered spaces', Proc. Nat. Acad. Sci. U.S.A. 26 (1940), 29-31.

[10] F. F. Bonsall and J. Duncan, Complete normed algebras, Springer-Verlag, 1973.

[11] N. Bourbaki, Integration, 2nd ed., Chapters 1-4, Hermann, Paris, 1965, Chapters 7 and $8,1963$.

[12] J. K. Brooks and P. W. Lewis, 'Linear operators and vector measures', Trans. Amer. Math. Soc. 192 (1974), 139-162.

[13] J. K. Brooks and N. Dinculeanu, 'Lebesgue-type spaces for vector integration, Linear operators weak completeness and weak compactness', J. Math. Anal. Appl. 54 (1976), 348-389.

[14] U. Cattaneo, 'On Mackey's imprimitivity theorem', Comment. Math. Helv. 54 (1979), 629-641.

[15] R. R. Christian, 'On order-preserving integration', Trans. Amer. Math. Soc. 86 (1957), 463-488. 
[16] R. Cristescu, 'Integrali vettoriali su uno spazio localmente compatto', Rend. Accad. Naz. Lincei, Roma Ser. VIII, 40 (1966), 792-795.

[17] R. Cristescu, Ordered spaces and linear operators, Bucuresti Ed. Academiei, Abacus Press, Kent, England, 1976.

[18] E. B. Davies, 'On the Borel structure of $C^{*}$-algebras', Comm. Math. Phys. 8 (1968), 147-163.

[19] E. B. Davies, 'On the repeated measurement of continuous observables in quantum mechanics', J. Funct. Anal. 6 (1970), 318-346.

[20] E. B. Davies and J. T. Lewis, 'An operational approach to quantum probability', Comm. Math. Phys. 17 (1970), 239-260.

[21] J. J. Diestel and J. J. Uhl, Jr., Vector measures, Math. Surveys, No. 15, Amer. Math. Soc., Providence, R. I., 1977.

[22] N. Dinculeanu, Vector measures, Pergamon Press, New York, 1967.

[23] J. Dixmier, Les algèbres d'opérateurs dans l'espace Hilbertien, 2ième éd., GauthierVillars, Paris, 1969.

[24] J. Dixmier, Les $C^{*}$-algèbres et leurs représentations, 2ième éd., Gauthier-Villars, Paris, 1969.

[25] I. Dobrakov, 'On integration in Banach spaces. I, II', Czechoslovak Math. J. 20 (95) (1970), 511-536, 680-695.

[26] - , 'On representation of linear operators on $C_{0}(T, X)$ ', Czechoslovak Math. J. 21 (1971), 13-30.

[27] N. Dunford, 'A bilinear integral I', Adv. in Math. 17 (1975), 337-342.

D8] R. J. Easton and D. H. Tucker, 'A generalized Lebesgue-type integral', Math. Ann. 181 (1969), 311-324.

[29] C. M. Edwards, 'The operational approach to algebraic quantum theory I', Comm. Math. Phys. 16 (1970), 207-230.

[30] C. M. Edwards, 'Classes of operations in quantum theory', Comm. Math. Phys. 20 (1971), 26-56.

[31] J. R. Edwards and S. G. Wayment, 'A unifying representation theorem', Math. Ann. 187 (1970), 317-328.

[32] E. G. Effros and E. Stermer, 'Jordan algebras of self-adjoint operators', Trans. Amer. Math. Soc. 127 (1967), 313-316.

[33] G. M. Fichtengolts and L. V. Kantorovich, 'Sur les opérations linéaires dans l'espace des fonctions', Studia Math. 5 (1935), 70-98.

[34] J. A. Goldstein, Semigroups of linear operators and applications, Oxford Univ. Press, Clarendon Press, 1985.

[35] R. K. Goodrich, 'A Riesz representation theorem', Proc. Amer. Math. Soc. 24 (1970), 629-636.

[36] A. Hartkamper and H. Neumann (eds), Foundations of quantum mechanics and ordered linear spaces, Lecture Notes in Physics, vol. 29, Springer-Verlag, 1974.

[37] L. V. Kantorovich, B. Z. Vulikh and A. G. Pinsker, Functional analysis in partially ordered spaces, Gostekhizdat, 1950 (Russian).

[38] L. V. Kantorovich and B. Z. Vulikh, 'Sur la représentation des opérations linéaires', Compositio Math. 5 (1937), 119-165.

[39] D. A. Kappos, Probability algebras and stochastic spaces, Academic Press, New York and London, 1969.

[40] J. L. Kelley, I. Namioka and co-authors, Linear topological spaces, Van Nostrand, Princeton, N. J., 1963.

[41] S. S. Khurana, 'Lattice-valued Borel measures', Rocky Mountain J. Math. 6 (1976), 377-382.

[42] I. Kluvanek, 'Operator valued measures and perturbations of semigroups', Arch. Rational Mech. Anal. 81 (1983), 161-180.

[43] I. Kluvanek, 'Integration and the Feynman-Kac formula', Studia Math. 87 (1987), $35-57$. 
[44] Ja. H. Kuckarov, 'Convergence of semifield-valued measures in the Prohorov metric', Dokl. Akad. Nauk SSSR 237 (2) (1977), 260-263 (Russian). English translation, Soviet Math. Dokl. 18 (6) (1977), 1415-1418.

[45] Ja. H. Kucharov, 'Convergence of semifield-valued distributions and a generalization of the Lindeberg-Feller theorem', Dokl. Akad. Nauk SSSR 237 (6) (1977), 1273-1276 (Russian). English translation, Soviet Math. Dokl. 18 (6) (1977), 1540-1544.

[46] S. Mazur and W. Orlicz, 'Sur les espaces métriques linéaires. I, II', Studia Math. 10 (1948), 184-208, ibid. 13 (1953), 137-179.

[47] E. J. McShane, Order-preserving maps and integration process, Ann. of Math. Studies 31, Princeton Univ. Press, Princeton, N. J., 1953.

[48] P. M. Morse and H. Feshbach, Methods of theoretical physics, McGraw-Hill, New York, 1953.

[49] F. Papangelou, 'Order convergence and topological completion of commutative latticegroups', Math. Ann. 155 (1964), 81-107.

[50] P. K. Pavlakos, 'On integration in partially ordered groups', Canad. J. Math. 35 (2) (1983), 353-372.

[51] P. K. Pavlakos, 'Convolutions and products of partially ordered vector-valued positive measures', Math. Ann., to appear.

[52] A. Peressini, Ordered topological vector spaces, Harper and Row, New York and London, 1967.

[53] A. Przeworska-Rolewicz and S. Rolewicz, 'On integrals of functions with values in a complete linear metric space', Studia Math. 26 (1966), 121-131.

[54] E. Rowecka, 'Random integrals and type and cotype of Banach space', Math. Z. 193 (1986), 381-391.

[55] S. Sakui, $C^{*}$-algebras and $W^{*}$-algebras, Springer-Verlag, Berlin and New York, 1971.

[56] T. A. Sarymsakov, B. A. Rubštein and V. I. Čilin, 'Complete tensor products of topological semifields', Dokl. Akad. Nauk SSSR 216 (6) (1974), 1226-1228 (Russian). English translation, Soviet Math. Dokl. 15 (3) (1974), 969-972.

[57] T. A. Sarymsakov, O. Ja. Benderskii and V. I. Čilin, 'Measures with values in semifields and their applications in probability theory', Dokl. Akad. Nauk SSSR 228 (1) (1976), 41-44 (Russian). English translation, Soviet Math. Dokl. 17 (3) (1976), 656-659.

[58] T. A. Sarymsakov, Semifields and probability theory, Lecture Notes in Math., vol. 550, Springer-Verlag, Berlin and New York, 1976, pp. 525-549.

[59] T. A. Sarymsakov and M. S. Gol' dštein, 'On partially ordered involutory algebras', Dokl. Akad. Nauk SSSR 228 (2) (1976), 306-309 (Russian). English translation, Soviet Math. Dokl. 17 (3) (1976), 725-729.

[60] T. A. Sarymsakov, Ja. H. Kučkarov and T. E. Dauletbaev, 'Convergence of Boolean measures in metric spaces', Dokl. Akad. Nauk SSSR 232 (5) (1977), 1023-1025 (Russian). English translation, Soviet Math. Dokl. 18 (1) (1977), 206-208.

[61] T. A. Sarymsakov, 'Noncommutative probability spaces on $O^{*}$-algebras', Dokl. Akad. Nauk SSSR 241 (2) (1978), 297-300 (Russian). English translation, Soviet Math. Dokl. 19 (4) (1978), 855-858.

[62] T. A. Sarymsakov and I. B. Halikulov, 'On the strong law of large numbers relative to semifield-valued measures', Dokl. Akad. Nauk SSSR 238 (4) (1978), 808-810 (Russian). English translation, Soviet Math. Dokl. 19 (1) (1978), 116-118.

[63] H. H. Schaefer, Topological vector spaces, Macmillan, New York, 1966.

[64] A. H. Shuchat, 'Integral representation theorems in topological vector spaces', Trans. Amer. Math. Soc. 172 (1972), 373-397.

[65] E. Størmer, 'On the Jordan structure of $C^{*}$-algebra', Trans. Amer. Math. Soc. 120 (1965), 438-447.

[66] E. Størmer, 'Jordan algebras of type I', Acta Math. 115 (1966), 165-184.

[67] E. Størmer, 'Irreducible Jordan algebras of self-adjoint operators', Trans. Amer. Math. Soc. 130 (1968), 153-166. 
[68] K. Swong, 'A representation theory of continuous linear maps', Math. Ann. 155 (1964), 270-291; errata, ibid. 157 (1964), 178.

[69] H. Tanabe, Equations of evolution, Pitman Press, 1979.

[70] D. Topping, 'Jordan algebras of self-adjoint operators', Mem. Amer. Math. Soc. 53 (1965).

[71] L. N. Tsitsas, 'Integral representations of linear maps', Bull. Soc. Math. Gréce (N.S.) 6 II, fasc. 2 (1965), 298-355.

[72] D. H. Uherka, 'Generalized Stieltjes integrals and a strong representation theorem for continuous linear maps on a function space', Math. Ann. 182 (1969), 60-66.

[73] H. Umegaki and A. T. Bharucha-Reid, 'Banach space-valued random variables and tensor products of Banach spaces', J. Math. Anal. Appl. 31 (1970), 49-67.

[74] B. Z. Vulikh, Introduction to the theory of partially ordered spaces (Fizmatgiz, Moscow, 1961). English translation, Noordhoff, Groningen, 1967.

[75] A. W. Wickstead, 'Stone-algebra-valued measures: Integration of vector-valued functions and Radon-Nikodym type theorems', Proc. London Math. Soc. 45 (3) (1982), 193-226.

[76] J. D. M. Wright, 'Stone-algebra-valued measures and integrals', Proc. London. Math. Soc. 19 (1969), 107-122.

[77] J. D. M. Wright, 'Vector-lattice measures on locally compact spaces', Math. Z. 120 (1971), 193-203.

[78] J. D. M. Wright, 'Measures with values in a partially ordered vector space', Proc. London Math. Soc. 25 (1972), 655-688.

[79] J. D. M. Wright, 'Products of positive vector measures', Quart. J. Math. (2) 24 (1973), 189-206.

[80] J. D. M. Wright, 'On minimal $\sigma$-completions of $C^{*}$-algebras', Bull. London Math. Soc. 6 (1974), 168-174.

[81] J. D. M. Wright, 'Regular $\sigma$-completions of $C^{*}$-algebras', J. London Math. Soc. 45 (2) (1976), 299-309.

[82] J. D. M. Wright, 'Jordan $C^{*}$-algebras', Michigan Math. J. 24 (1977), 291-302.

[83] K. Yosida, Functional analysis, 6th ed., Springer-Verlag, Berlin and New York, 1980.

\section{Department of Mathematics}

University of Athens

Athens

Greece 